\title{
Fatigue Detection Method based on Common Spatial Subspace Decomposition
}

\author{
Mu Zhendong \\ College of Information Engineering, Jiangxi College of Technology, Jiangxi Nanchang 330098
}

Keywords: Fatigue detection; Common Spatial Subspace Decomposition; EEG

\begin{abstract}
Fatigue has been a common problem in modern society, it is constantly eroding the people's health, and there is not an effective method for fatigue testing. Based on the brain waves are analyzed using methods Common Spatial Subspace Decomposition fatigue analysis to determine a clear value as a reference, other acquisition of EEG and the extent of this reference comparison, the final calculation of fatigue, by people at different times of monitoring, data showed that the extent of the reaction can be human fatigue to some extent.
\end{abstract}

\section{Introduction}

With the quickening pace of society, people's living standards continue to improve at the same time, people's health status is increasingly worse, there is fatigue in one of the factors affecting people's health, often seen on the internet, there's a young staff due to fatigue died, but people do not have a valid means to monitor the person's fatigue.

With the in-depth study of EEG, more and more research shows that by monitoring brain waves can be detected by human fatigue, but this research study were performed in the laboratory of a state, how to detect fatigue and displayed in the form of value, there is no effective method.

By Common Spatial Subspace Decomposition methods to achieve two states characterized by comparison to the waking state standards to collect the EEG as an object, to achieve the ratio between the two as a basis to measure fatigue, the successful solution of this problem.

\section{Method}

Common Spatial Subspace Decomposition(CSSD), Need to design experiments for comparison of two groups, respectively comprising the activities caused by different tasks, and also contains the same task-independent background activity. By comparing the two sets of data, you can put the task-induced activity patterns extracted.

Data Matrix Let A and B are two tasks were achieved under $X_{A}$ and $X_{B}$, they are written in the form of temporal source model

$$
\underset{N \times T}{X_{A}}=\left[C_{a} C_{c}\right]\left[\begin{array}{c}
S_{a} \\
S_{c}^{A}
\end{array}\right] \quad \underset{N \times T}{X_{B}}=\left[C_{b} C_{c}\right]\left[\begin{array}{c}
S_{b} \\
S_{c}^{B}
\end{array}\right]
$$


Here, $\mathrm{N}$ is the number of channels, $\mathrm{T}$ is the sampling points; $C_{a}$ only contains the $m_{a}$ task A related EEG spatial mode, $C_{b}$ contains the $m_{b}$ only task B related space mode. $C_{c}$ contains $m_{c}$ simultaneous with $\mathrm{A}$ and $\mathrm{B}$ associated mode. $S_{a}, S_{b}, S_{c}^{A}, S_{c}^{B}$ is the corresponding source activities. Number Without loss of generality, it is assumed linearly independent source does not exceed the number of channels, namely.

$$
m_{a}+m_{b}+m_{c} \leq N
$$

CSSD is calculated as follows:

1, calculate the covariance matrix of $X_{A}$ and $X_{B}$ :

$$
R_{A}=X_{A} X_{A}^{\prime}, R_{B}=X_{B} X_{B}^{\prime}
$$

2 , set $R=R_{A}+R_{B}$, a primary component of the decomposition of the $\mathrm{R}$

$$
R=U_{0} \sum U_{0}^{\prime}
$$

Define $P=\sum^{-1 / 2} U_{0}^{\prime}, \quad W=U_{0} \sum^{1 / 2}$

3, set $S_{A}=P R_{A} P^{\prime}, S_{B}=P R_{B} P^{\prime}$. And then decide on the $S_{A}$ and $S_{B}$ component decomposition.

$$
S_{A}=U_{A} \sum_{A} U_{A}^{\prime}, \quad S_{B}=U_{B} \sum_{B} U_{B}^{\prime}
$$

4, For tasks A, U out the first $m_{a}$ columns, denoted $U_{a}$, define $S P_{a}=W U_{a}, S F_{a}=U_{a}^{\prime} P$, and then signal A component of the task can be estimated:

$$
\widehat{X}_{A}=S P_{a} S F_{a} X_{A}
$$

\section{Result}

The EEG data used in this paper is the BCI Laboratory of Jiangxi Institute of technology through the EEG acquisition of College students. EEG acquisition is the use of 40-Neuroscan amplifier, were obtained by scan 4.3 software, the reference electrode by way of the right mastoid as reference electrode, using $1000 \mathrm{~Hz}$ sampling rate, band acquisition using $200 \mathrm{~Hz}$ low-pass, high-pass $0.05 \mathrm{~Hz}$ and $50 \mathrm{~Hz}$ notch. Each experiment were collected continuously for 5 minutes, each subject every 4 experiment.

Respectively, 20 subjects were collected EEG experiments, through artificial selection, each subject section 600 can be used to select the available EEG, which under section 300 as a quiet state, under section 300 as fatigue, EEG after filtering the initial processing, the fatigue calculation using the above methods.

Fatigue calculation results shown in Figure 1: 


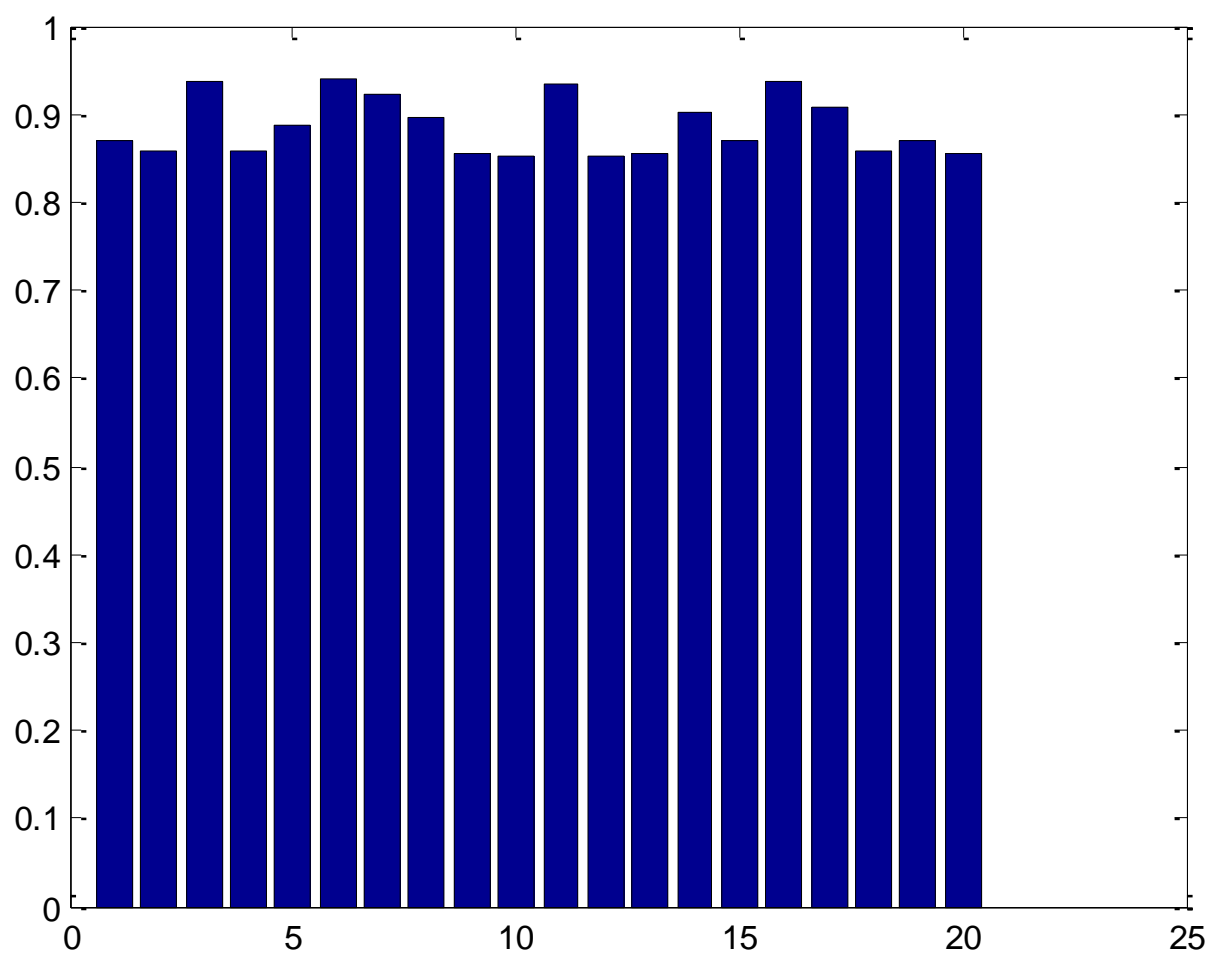

Figure. 1 Fatigue calculation accuracy.

$\mathrm{X}$-axis in Figure 1 represents an individual subject; $\mathrm{y}$-axis represents the EEG for 300 fatigue calculation accuracy.

\section{Acknowledgements}

This work was financially supported by project of Technology Department of Jiangxi Province [No 20143BBM26048] and project of Jiangxi University of Technology [No. xtcx201312].

\section{References}

[1] Ji Qiang, Yang Xiaojie. "Real-Time Eye, Gaze, and Face Pose Tracking for Monitoring Driver Vigilance". Real-Time Imaging, August, 2002, pp.357-377

[2] Strijkstra AM, Beersma DG, Drayer B, Halbesma N, Daan S. "Subjective sleepiness correlates negatively with global alpha $(8-12 \mathrm{~Hz})$ and positively with central frontal theta $(4-8 \mathrm{~Hz})$ frequencies in the human resting awake electroencephalogram". Neurosci Lett, Vol. 340, No.1, 2003, pp. 17-20.

[3] Shi Licheng, Yu Hong, Lu Baoliang. "Semi-Supervised Clustering for Vigilance Analysis Based on EEG”. Intern J Conference on Neural Network, Orlando, US, Aug. 2007, pp. 1518-1523.

[4] Yu Hong, Shi Licheng, Lu Baoliang. "Vigilance Estimation Based on EEG Signals". IEEE/ICME International Conference on Complex Medical Engineering (CME2007), May, 2007.

[5] Angelakis E, Hatzis A, Panourias IG, Sakas DE. "Brain-computer interface: a reciprocal self-regulated neuromodulation”. Acta Neurochir Suppl. Vol. 97, 2007, pp.555-9. 
[6] Bayliss JD, Inverso SA, Tentler A. “Changing the P300 brain computer interface”. Cyberpsychol Behav., Vol.7, No.6, 2004, pp.694-704.

[7] Bianchi L, Quitadamo LR, Garreffa G, Cardarilli GC, Marciani MG. "Performances evaluation and optimization of brain computer interface systems in a copy spelling task". IEEE Trans Neural Syst Rehabil Eng. Vol.15, No.2, 2007, pp.207-16.

[8] Wu Shao-bin,Gao Li,Wang Liu-an, "Detectin Driving Fatigue Based On electroencephalogram”, Transactions of Beijing Institute of Technology, Vol. 29,No 12, 2009 , pp1072-1075

[9] Li Mingai, Zhang Cheng, Yang Jinfu, "EEG-Based Method to Determine the Drowsiness Degree of EEG signal”, Beijng Biomedical Engneering, Vol. 30 No1, 2011， pp 57-62 